\title{
BMJ Open Maternal and birth cohort studies in the Gulf Cooperation Council countries: protocol for a systematic review and narrative evaluation
}

\author{
Rami H. Al-Rifai, ${ }^{1}$ Nasloon Ali, ${ }^{1}$ Esther T. Barigye, ${ }^{1}$ Amal H. I. Al Haddad, ${ }^{1}$ \\ Tom Loney, ${ }^{1,2}$ Fatima Al-Maskari, ${ }^{1}$ Luai A. Ahmed ${ }^{1}$
}

To cite: Al-Rifai RH., Ali N, Barigye ET., et al. Maternal and birth cohort studies in the Gulf Cooperation Council countries: protocol for a systematic review and narrative evaluation. BMJ Open 2018;8:e019843. doi:10.1136/ bmjopen-2017-019843

\section{- Prepublication history and} additional material for this paper are available online. To view these files, please visit the journal online (http://dx.doi. org/10.1136/bmjopen-2017019843).

Received 2 0ctober 2017 Revised 16 November 2017 Accepted 11 December 2017

Check for updates

${ }^{1}$ College of Medicine and Health Sciences, Institute of Public Health, United Arab Emirates University, Al Ain, United Arab Emirates

${ }^{2}$ College of Medicine, Mohammed Bin Rashid University of Medicine and Health Sciences, Dubai, United Arab Emirates

Correspondence to

Dr Rami H. Al-Rifai;

rrifai@uaeu.ac.ae

\section{ABSTRACT}

Introduction Cohort studies have revealed that genetic, socioeconomic, communicable and noncommunicable diseases, and environmental exposures during pregnancy may influence the mother and her pregnancy, birth delivery and her offspring. Numerous studies have been conducted in the Gulf Cooperation Council (GCC) countries to examine maternal and birth health. The objectives of this protocol for a systematic review are to systematically review and characterise the exposures and outcomes that have been examined in the mother and birth cohort studies in the GCC region, and to summarise the strength of association between key maternal exposures during pregnancy (ie, body mass index) and different health-related outcomes (ie, mode of birth delivery). The review will then synthesise and characterise the consequent health implications and will serve as a platform to help identify areas that are overlooked, point out limitations of studies and provide recommendations for future cohort studies.

Methods and analysis Medline, Embase, Cochrane Library and Web of Science electronic databases will be comprehensively searched. Two reviewers will independently screen each study for eligibility, and where discrepancies arise they will be discussed and resolved; otherwise a third reviewer will be consulted. The two reviewers will also independently extract data into a predefined Excel spreadsheet. The included studies will be categorised on the basis of whether the participant is a mother, infant or mother-infant dyad. Outcome variables will be divided along two distinctions: mother or infant. Exposure variables will be divided into six domains: psychosocial, biological, environmental, medical/medical services, maternal/ reproductive and perinatal/child. Studies are expected to be of heterogeneous nature; therefore, quantitative syntheses might be limited.

Ethics and dissemination There is no primary data collection; therefore, ethical review is not necessary. The findings of this review will be disseminated in a peer-reviewed journal and presented at relevant conferences.

PROSPERO registration number CRD42017068910.
Strengths and limitations of this study

- This systematic review will provide a comprehensive appraisal of the mother and birth cohort studies that have been conducted in the Gulf Cooperation Council (GCC) region using a robust search strategy and assessment methodology.

- The results of this systematic review will summarise the limitations and identify gaps in mother and birth cohort studies that have been conducted in the GCC region. This may help researchers to avoid the identified limitations and address gaps in future cohort studies on mother and birth health.

- In view of the anticipated heterogeneity in effect estimates between different measured exposures and outcomes, it may be difficult to conduct meta-analyses. Included studies may have little in common due to clinical (eg, different populations), methodological (eg, retrospective vs prospective) and analytical heterogeneity, as well as different exposures and outcomes.

\section{INTRODUCTION}

Evidence has shown that genetic, socioeconomic status (SES) and environmental exposures during pregnancy have resultant outcomes starting in pregnancy, during birth delivery, in the life of an infant and can have long-term consequences in the child's later life. ${ }^{1-5}$ These outcomes may be related to cardiovascular diseases (CVD), cancers, respiratory diseases, cognitive decline or psychological health. ${ }^{67}$ Gestational diabetes increases the risk of the mother developing postpregnancy type 2 diabetes mellitus ${ }^{8}$ and increases the risk of macrosomia in the newborn. ${ }^{9}$ Maternal obesity and gestational weight gain are independent predictors of macrosomia in newborns. ${ }^{9}$ Pregnant women experiencing pre-eclampsia are at a greater risk of developing CVD (peripheral artery disease, ischaemic heart disease, myocardial infarction or cardiac shock) in their later stages of life..$^{10-12}$ 
Early-onset pre-eclampsia can lead to an increased risk of developing metabolic syndrome compared with lateonset pre-eclampsia. ${ }^{13}$ Furthermore, pre-eclampsia is associated with an increased risk of elevated pulmonary artery pressure and reduced flow-mediated dilation, ${ }^{14}$ and doubles the risk of stroke in offspring. ${ }^{15}$ Maternal race and education, ${ }^{16}$ poverty and level of neighbourhood SES, ${ }^{17-19}$ and exposure to air pollutants may have adverse pregnancy outcomes, including low birth weight and preterm delivery. ${ }^{20}$ These factors could individually or jointly adversely affects maternal and birth outcomes.

Evidence has shown that urbanisation and economic progress in emerging economies have led to a drastic reduction in physical activity with a concomitant rise in chronic diseases. ${ }^{21}{ }^{22}$ The Gulf Cooperation Council (GCC) includes Bahrain, Kuwait, Oman, Saudi Arabia, United Arab Emirates and Qatar. Given the rich oil and gas reserves in the GCC region, these countries have experienced a unique economic development that is associated with a rapid transition from agrarian to industrialisation. ${ }^{23}$ This shift is associated with changes in demographic dynamics and composition of the population. Dramatic lifestyle-related changes have occurred over the last few decades. The diet in the GCC region has shifted from relying on the traditional high-fibre, low-fat Arab diet to low-fibre, high-fat and high-sugar Westernised diet, with low intake of fruits and vegetables. ${ }^{24}$ The prevalence of physical inactivity and tobacco smoking is very high. Although tobacco consumption is not as frequent among women, hookah (water pipe) smoking is increasingly common among both genders in the GCC region. ${ }^{25}$ Along with these lifestyle changes, there is a dramatic rise in the prevalence of several health outcomes including obesity, diabetes mellitus, asthma, neurodevelopmental disorders and CVD. ${ }^{25}{ }^{26}$ Therefore, the emerging risks to maternal and prenatal health have required obtaining locally relevant data on which to base more effective public health policies.

Adverse health trajectories established in early life may have long-term consequences. Many maternal and prenatal exposures and outcomes have been studied in the GCC region. ${ }^{27-29}$ Moreover, there is an emerging body of multidimensional research in the GCC region that has captured mother and birth exposures including anthropometric, SES, environmental, physiological, lifestyle, biochemical and medical exposures, ${ }^{30} 31$ and consequently the resulting outcomes such as the circumstances of pregnancy, delivery, neonatal and perinatal growth and health. ${ }^{32-35}$ However, these studies are yet to be collated, summarised, criticised and discussed. Further investigation of the literature is needed to explore the role of prenatal exposures on maternal and neonatal health-related outcomes in the GCC region.

This systematic review will characterise exposures measured and assessed in pregnant women in the GCC region and describe the association between the measured exposures and the different maternal and birth health-related outcomes. The review will provide the first synthesis of maternal, neonatal and prenatal risk factors and outcomes in the GCC region; provide an overview of the breadth of variables studied and the study methodology used; and identify the consequent health implications. This review will support an understanding of which aspects of a pregnant mother and infant are unique to specific contexts in the GCC Arab population and which are universal to all populations in different countries. Areas and topics that are relevant to the region but are overlooked or understudied will be identified, and we will allude to the limitations in the included studies and offer recommendations for future researches.

\section{Aim}

The aim of this study is to systematically review mother and birth cohort studies that have been conducted in the GCC region and chronicle the exposures and outcomes that have been examined and discussed. If sufficient data are available, this study will also aim to provide a quantitative summary on the prevalence of exposures related to a specific outcome(s), as well as the strength of association between exposure and health-related outcome(s) in both mothers and their offspring. The systematic review will serve as a platform that would help in identifying areas and topics that are overlooked, point out limitations of studies and offer recommendations for future cohort studies.

\section{Objectives}

To achieve the above aim, the following will be the review objectives:

1. summarise and characterise all mother and birth cohort studies conducted in the GCC region

2. summarise the strength of association between maternal exposures during pregnancy (ie, body mass index) and different maternal and birth health-related outcomes (ie, birth weight and mode of birth delivery).

\section{METHODS AND ANALYSIS \\ Study design}

This protocol is registered online on PROSPERO, the International Prospective Register of Systematic Reviews (http:www.crd.york.ac.uk/PROSPERO, trial registration number CRD42017068910). Following the Preferred Reporting Items for Systematic Review and Meta-analysis Protocols (PRISMA-P) 2015 checklist, ${ }^{36}$ a systematic review of the literature will be performed to identify mother and birth cohort studies that have been reported in the GCC region and have examined different early exposures and maternal and birth related-health outcomes. Please refer to the PRISMA-P checklist in online supplementary table S1.

\section{Studies inclusion and exclusion criteria}

We will include mother and birth cohort studies (prospective or retrospective) conducted in the GCC region countries regardless of the exposures and outcomes measured or assessed. Mother and birth cohort studies should 
have recruited pregnant mothers and their offspring or recruited offspring immediately after delivery as long as relevant information on pregnancy is available. In a sense, cohort studies should have followed up both mothers and their offspring and measured different maternal exposures and maternal and newborn outcomes. These studies must have been published by the search date of November 2017 with no restriction on the language.

Potentially duplicate research reports will be compared using the date of publication and publication year, country where the study was conducted, number of participants and number of subjects enrolled. In the case of duplicate publications, only one article with more comprehensive information will be included in the review. Cohort studies describing only exposed with no comparison group, or studies other than cohort designs (eg, cross-sectional, case-control) or in countries other than the GCC region will be excluded.

\section{Search strategy for identification of relevant studies \\ Data sources}

Electronic databases will include Medline, Embase, Cochrane Library and Web of Science.

\section{Hand-searching}

All reference lists of retrieved articles will be scanned for further studies.

\section{Search strategy}

A comprehensive electronic and sensitive search strategy using variant search terms that are tailored to suit the individual database search requirements will be used to identify relevant cohort studies in the GCC region. We will combine the search terms using the Boolean operator 'AND', while the keywords within each group will be linked using the operator 'OR'. Database search strategies are presented in the box. The initial selection criteria will be broad to ensure that as many studies as possible are assessed based on their relevance to the review. Any articles that are not relevant to the study will be excluded in the early stages of the titles and abstracts screening after de-duplication. The full-text articles of seemingly relevant titles/abstracts will be accessed online. Those that are not freely available online will be obtained through the University Interlibrary Loan System. The full articles will be read for further screening based on the eligibility criteria.

\section{Data extraction}

Data from the eligible studies will be extracted into a predefined Excel spreadsheet, which will first be piloted using five studies. Data will be extracted independently by two investigators. The two investigators will compare and discuss their findings to resolve any differences. Data to be extracted from the relevant studies will include author names, date of publication, country where the study was conducted, number of participants, publication year, study design, country, setting, target population, subject/ participant, study period, follow-up period, measuring
Box Data sources and search criteria for systematically reviewing literature reporting on maternal and birth cohort studies in the Gulf Cooperation Council countries

Databases search protocol

Filters: no language restrictions, title/abstract, humans.

\section{Medline via PubMed}

cohort[MeSH Terms] AND \&(((((pregnancy) OR pregnancy complications) OR maternal exposure) $O R$ infant) $O R$ child) $O R$ fetus)) AND ()((()(((bahrain[MeSH Terms]) OR kuwait[MeSH Terms]) OR oman[MeSH Terms]) OR qatar[MeSH Terms]) OR saudi arabia[MeSH Terms]) OR united arab emirates[MeSH Terms]))))

\section{Embase}

('cohort':ab,ti AND pregnan*:ab,ti AND complication*:ab,ti OR maternal:ab,ti) AND (exposure*:ab,ti OR infant*:ab,ti OR child*:ab,ti OR fetus*:ab,ti) AND ('bahrain' OR 'kuwait' OR 'oman' OR 'qatar' OR 'saudi arabia' OR 'united arab emirates')

\section{Web of Science}

(TS=cohortAND TI=(Pregnan* OR Pregnan* Complication* OR Mater* Exposur* OR Infant* OR Child* OR Fetus*) AND CU=('Bahrain' OR 'Kuwait' OR 'Oman' OR 'Qatar' OR 'Saudi Arabia' OR 'United Arab Emirates'))

\section{Cochrane Library}

(Cohort AND (Pregnancy OR Pregnancy Complications OR Maternal Exposure OR Infant OR Child OR Fetus) AND ('bahrain' OR 'kuwait' OR 'oman' OR 'qatar' OR 'saudi arabia' OR 'united arab emirates'))

tools used for the mother, measuring tools used for the infant, number of subjects eligible or expected, number of subjects enrolled, number of subjects at follow-up, and exposure and outcome variables. The full list of variables to be extracted is available in online supplementary table S2.

\section{Data synthesis and analysis \\ Data assessment}

The studies will be categorised into different domains based on the exposures and outcomes that were measured and assessed. Eligible studies will be categorised on the basis of whether the participant is a mother, infant or mother-infant dyad. Outcome variables will be divided into mother or infant. Exposure variables will be divided into six domains: psychosocial (ie, depression, education, employment), biological including genetically inherited illnesses (ie, glucose-6-phosphate dehydrogenase deficiencies), environmental (ie, exposure to fuels or secondhand smoke), medical (ie, diabetes mellitus, hypertension), medical services (ie, length of waiting times to receive healthcare, relationships with the healthcare professionals), maternal/reproductive (ie, gestational diabetes, breast feeding) and perinatal/child (ie, birth weight, multiplicity, mode of birth delivery). Five of these domains will pertain to the mother, and only one domain of perinatal/child will pertain to the infant. The perinatal/child exposure domain will not be subcategorised. Medical/medical services exposures will refer to pre-existing conditions of the mother that are not due to 
pregnancy. Maternal/reproductive exposures will refer to conditions that are caused by the pregnancy.

\section{Synthesis}

Studies are expected to be of a heterogeneous nature, and therefore, initially a narrative synthesis method will be employed. The synthesis of the type of data collected will be divided according to maternal exposures such as SES, perinatal and infant exposures such as eclampsia and pre-eclampsia, and maternal outcomes such as mode of birth delivery, and fetal outcomes such as gestational age, stillbirth and birth weight. Meta-analyses summarising the pooled prevalence of different exposures (eg, SES) on different maternal and birth health-related outcomes (eg, birth weight and mode of birth delivery) and to quantitatively summarise the strength of association between different exposures on different maternal and child health-related outcomes will be performed. Conducting meta-analyses will depend on the availability of sufficient data (three effect estimates or more) and using the random-effects model. Pooling effect estimates on the association between key exposure-outcome dyads will be according to the type of the reported effect estimate. Crude estimates will be pooled independent of the adjusted estimates. Effect estimates in the form of ORs will be pooled independent of estimates reported in the form of relative risks, rate ratios or HRs. We will assess heterogeneity between studies using Cochran's $Q$ statistic and I-squared $\left(I^{2}\right)$ tests. We will use the $I^{2}$ statistic to estimate the percentage of total variation across studies due to true between-study differences rather than chance. Sources of heterogeneity will be explored through subgroup analysis using study-level characteristics such as age groups, study period, year of publication and sample sizes. This will be complemented where relevant by meta-regression to further explain the sources of heterogeneity, if any.

\section{Quality and risk of bias assessment}

Two reviewers will independently screen each title and abstract for relevance and potential eligibility for fulltext assessment. The two reviewers will then compare the selected studies. Discrepancies will be discussed and resolved to reach a consensus; otherwise a third reviewer will be used to review the abstract and determine inclusion. The second screening will be for the full articles of seemingly relevant titles and abstracts. These will also be independently screened in detail by the two reviewers. Similarly, where the reviewers disagree, consensus will be reached by discussion or consultation with the third reviewer. Methodological aspects of the included cohort studies will be assessed for quality using a validated quality appraisal tool. ${ }^{37}$ Cohort studies will be categorised into low or high risk of bias (ROB) based on six quality assessment domains: (1) whether exposed and non-exposed cohorts were recruited from the same population; (2) confidence in the assessment of exposure; (3) confidence in the assessment of outcome; (4) the outcome of interest was not present at the start of the study; (5) matching of exposed and unexposed cohorts in key potential confounders (eg, age); and (6) whether the follow-up of cohorts was adequate. The answer to each domain will be either 'yes=low ROB', 'No=high ROB' or 'unclear=ROB is an unclear'. Unclear ROB category will be assigned for studies with missing information for any specific domain of the six quality assessment domains. The overall quality of evidence on the exposure and outcome might be assessed using the Grading of Recommendations Assessment, Development and Evaluation tool. ${ }^{38}$

\section{Assessing quality of evidence}

We will assess meta-bias through assessing the presence of publication bias by generating funnel plots. Funnel plots will be generated for data included in the meta-analyses supplemented with formal statistical testing using the Egger's ${ }^{39}$ and the Begg's ${ }^{40}$ tests for publication bias, whenever sufficient data are available.

\section{Ethics and dissemination}

The findings of this review will be disseminated in a peerreviewed journal and presented at relevant conferences.

Contributors RHA, NA, ETB, AHIAH, TL, FAM and LAA conceptualized and designed the protocol. NA and ETB drafted the manuscript. RHA, NA, ETB, AHIAH, TL, FAM and LAA critically revised the manuscript. RHA, NA, ETB, AHIAH, TL, FAM and LAA approved the final submission of the manuscript. RHA is the guarantor of the protocol.

Funding This protocol received no specific funding. We greatly thankful for the infrastructure provided by the Institute of Public Health at the College of Medicine and Health Sciences, United Arab Emirates University.

Disclaimer The protocol has been developed in conjunction of the seven authors who are all employed and salaried staff by the United Arab Emirates University.

Competing interests None declared.

Patient consent Not required.

Ethics approval There are no primary data to be collected for this review; therefore, ethical review is not necessary.

Provenance and peer review Not commissioned; externally peer reviewed.

Open Access This is an Open Access article distributed in accordance with the Creative Commons Attribution Non Commercial (CC BY-NC 4.0) license, which permits others to distribute, remix, adapt, build upon this work non-commercially, and license their derivative works on different terms, provided the original work is properly cited and the use is non-commercial. See: http://creativecommons.org/ licenses/by-nc/4.0/

(c) Article author(s) (or their employer(s) unless otherwise stated in the text of the article) 2018. All rights reserved. No commercial use is permitted unless otherwise expressly granted.

\section{REFERENCES}

1. Correa C, Mallarino C, Peña R, et al. Congenital malformations of pediatric surgical interest: prevalence, risk factors, and prenatal diagnosis between 2005 and 2012 in the capital city of a developing country. Bogotá, Colombia. J Pediatr Surg 2014;49:1099-103.

2. Kim JH, Scialli AR. Thalidomide: the tragedy of birth defects and the effective treatment of disease. Toxicol Sci 2011;122:1-6.

3. Rogers I, Emmett P, Ness A, et al. Maternal fish intake in late pregnancy and the frequency of low birth weight and intrauterine growth retardation in a cohort of British infants. J Epidemiol Community Health 2004;58:486-92.

4. Kogan MD. Social causes of low birth weight. $J R$ Soc Med 1995;88:611-5.

5. Woodruff TJ, Parker JD, Kyle AD, et al. Disparities in exposure to air pollution during pregnancy. Environ Health Perspect 2003;111:942-6. 
6. Christian P, Stewart CP. Maternal micronutrient deficiency, fetal development, and the risk of chronic disease. J Nutr 2010;140:437-45.

7. Greenough A. Prenatal factors in the development of chronic lung disease. Semin Fetal Neonatal Med 2009;14:339-44.

8. Bellamy L, Casas JP, Hingorani AD, et al. Type 2 diabetes mellitus after gestational diabetes: a systematic review and meta-analysis. Lancet 2009;373:1773-9.

9. Alberico S, Montico M, Barresi V, et al. The role of gestational diabetes, pre-pregnancy body mass index and gestational weight gain on the risk of newborn macrosomia: results from a prospective multicentre study. BMC Pregnancy Childbirth 2014;14:23.

10. Lin YS, Tang CH, Yang CY, et al. Effect of pre-eclampsia-eclampsia on major cardiovascular events among peripartum women in Taiwan. Am J Cardiol 2011;107:325-30.

11. Kestenbaum B, Seliger SL, Easterling TR, et al. Cardiovascular and thromboembolic events following hypertensive pregnancy. Am J Kidney Dis 2003;42:982-9.

12. Hannaford P, Ferry $S$, Hirsch $S$. Cardiovascular sequelae of toxaemia of pregnancy. Heart 1997;77:154-8.

13. Stekkinger E, Zandstra M, Peeters LL, et al. Early-onset preeclampsia and the prevalence of postpartum metabolic syndrome. Obstet Gynecol 2009;114:1076-84.

14. Jayet PY, Rimoldi SF, Stuber T, et al. Pulmonary and systemic vascular dysfunction in young offspring of mothers with preeclampsia. Circulation 2010;122:488-94.

15. Kajantie E, Eriksson JG, Osmond C, et al. Pre-eclampsia is associated with increased risk of stroke in the adult offspring: the Helsinki birth cohort study. Stroke 2009;40:1176-80.

16. Gray SC, Edwards SE, Schultz BD, et al. Assessing the impact of race, social factors and air pollution on birth outcomes: a populationbased study. Environ Health 2014;13:4.

17. Weck RL, Paulose T, Flaws JA. Impact of environmental factors and poverty on pregnancy outcomes. Clin Obstet Gynecol 2008;51:349-59.

18. Généreux M, Auger N, Goneau M, et al. Neighbourhood socioeconomic status, maternal education and adverse birth outcomes among mothers living near highways. J Epidemiol Community Health 2008;62:695-700.

19. Luo ZC, Wilkins R, Kramer MS, et al. Effect of neighbourhood income and maternal education on birth outcomes: a population-based study. CMAJ 2006;174:1415-20.

20. Kent ST, McClure LA, Zaitchik BF, et al. Area-level risk factors for adverse birth outcomes: trends in urban and rural settings. BMC Pregnancy Childbirth 2013;13:129.

21. Ng SW, Norton EC, Popkin BM. Why have physical activity levels declined among Chinese adults? Findings from the 1991-2006 China Health and Nutrition Surveys. Soc Sci Med 2009;68:1305-14.

22. Monda KL, Gordon-Larsen P, Stevens J, et al. China's transition: the effect of rapid urbanization on adult occupational physical activity. Soc Sci Med 2007;64:858-70.
23. The World Bank. The World Bank in the Gulf Cooperation Council. http://www.worldbank.org/en/country/gcc/overview

24. al-Mahroos F, al-Roomi K. Overweight and obesity in the Arabian Peninsula: an overview. J R Soc Promot Health 1999;119:251-3.

25. Alshaikh MK, Filippidis FT, Al-Omar HA, et al. The ticking time bomb in lifestyle-related diseases among women in the Gulf Cooperation Council countries; review of systematic reviews. BMC Public Health 2017; $17: 536$

26. Rahim HF, Sibai A, Khader Y, et al. Non-communicable diseases in the Arab world. Lancet 2014;383:356-67.

27. Al Juaid DA, Binns CW, Giglia RC. Breastfeeding in Saudi Arabia: a review. Int Breastfeed J 2014;9:1.

28. Al-Essa M, Azad RV, Rashwan N. Rate of and Risk Factors Associated with Retinopathy of Prematurity: A Prospective Study from Kuwait. Med Principles Pract 1999;8:115-8.

29. Al Mahroos S, Nagalla DS, Yousif W, et al. A population-based screening for gestational diabetes mellitus in non-diabetic women in Bahrain. Ann Saudi Med 2005;25:129-33.

30. Al-Riyami IM, Al-Busaidy IQ, Al-Zakwani IS. Medication use during pregnancy in Omani women. Int J Clin Pharm 2011;33:634-41.

31. Dashti M, Scott JA, Edwards CA, et al. Predictors of breastfeeding duration among women in Kuwait: results of a prospective cohort study. Nutrients 2014;6:711-28.

32. Al-Mulhim AA, Abu-Heija A, Al-Jamma F, et al. Pre-eclampsia: maternal risk factors and perinatal outcome. Fetal Diagn Ther 2003;18:275-80

33. Wahabi HA, Esmaeil SA, Fayed A, et al. Pre-existing diabetes mellitus and adverse pregnancy outcomes. BMC Res Notes 2012;5:496.

34. Al Arfaj AS, Khalil N. Pregnancy outcome in 396 pregnancies in patients with SLE in Saudi Arabia. Lupus 2010;19:1665-73.

35. Al-Saleh I, Nester M, Mashhour A, et al. Prenatal and postnatal lead exposure and early cognitive development: longitudinal study in Saudi Arabia. J Environ Pathol Toxicol Oncol 2009;28:283-302.

36. Moher D, Shamseer L, Clarke M, et al. Preferred reporting items for systematic review and meta-analysis protocols (PRISMA-P) 2015 statement. Syst Rev 2015;4:1.

37. Viswanathan M, Berkman ND, Dryden DM, et al. Assessing risk of bias and confounding in observational studies of interventions or exposures: further development of the RTI Item Bank [Internet]. Rockville, MD: Agency for Healthcare Research and Quality (US), 2013. Report No: 13-EHC106-EF.

38. Guyatt GH, Oxman AD, Schünemann HJ, et al. GRADE guidelines: a new series of articles in the Journal of Clinical Epidemiology. J Clin Epidemiol 2011;64:380-2.

39. Egger M, Meta-Analysis SGD. Potentials and promise. BMJ 1997;315:1371-4.

40. Begg CB, Mazumdar M. Operating characteristics of a rank correlation test for publication bias. Biometrics 1994;50:1088-101. 